\title{
Asymptotic Behaviour of the Boltzmann Equation with Infinite Range Forces
}

\section{Leif Arkeryd}

Department of Mathematics, Chalmers University of Technology and University of Göteborg, S-412 96 Göteborg, Sweden

\begin{abstract}
We have previously obtained existence results for the space-homogeneous, non-linear Boltzmann equation for a class of encounters with infinite range, including inverse $k^{\text {th }}$ power molecules with $k>3$. In the present paper those solutions are proved to converge in weak $L^{1}$-sense for $k \geqq 5$ to Maxwellian distributions when $t \rightarrow \infty$. Also the higher moments converge to those of the relevant Maxwellian. The method of proof relies on non-standard techniques.
\end{abstract}

\section{Preliminaries}

Consider the space-homogeneous, non-linear Boltzmann equation in the absence of exterior forces

$$
D_{t} f(t, v)=Q f(t, v) \quad\left(t>0, v \in R^{3}\right)
$$

with Cauchy data

$$
f(0, v)=f_{0}(v) \geqq 0, \quad\left(v \in R^{3}\right) .
$$

Here $Q=Q_{k}$ denotes the collision operator

$$
Q f\left(v_{1}\right)=Q_{w} f\left(v_{1}\right)=\int_{R^{3} \times B^{2}}\left[f \otimes f\left(J_{u}\left(v_{1}, v_{2}\right)\right)-f \otimes f\left(v_{1}, v_{2}\right)\right] w\left(v_{1}, v_{2}, u\right) d v_{2} d u,
$$

and

$$
f \otimes g\left(v_{1}, v_{2}\right)=f\left(v_{1}\right) g\left(v_{2}\right) .
$$

In the cut-off case the impact parameter $u$ in $R^{2}$ is restricted to a disc

$$
B^{2}(r)=\left\{u \in R^{2} ;|u| \leqq r\right\} .
$$

In the present paper, however, with intermolecular forces of infinite range, we consider

$$
B^{2}=B^{2}(\infty)=R^{2} .
$$


The velocity after collision of two colliding particles with impact parameter $u$ and initial velocity $v_{1}$ and $v_{2}$ is given by

$$
\left(v_{1}^{\prime}, v_{2}^{\prime}\right)=J_{u}\left(v_{1}, v_{2}\right)
$$

The diffeomorphism $J$ is generated from $k^{\text {th }}$ power molecules with $k \geqq 5$, and the weight-function

$$
w\left(v_{1}, v_{2}\right)=\left|v_{1}-v_{2}\right|
$$

is the physical one, together with the cut-off version

$$
w_{n}\left(v_{1}, v_{2}, u\right)=\left\{\begin{array}{l}
w \text { for }|u| \leqq n, w \leqq n \\
n \text { for }|u| \leqq n, w>n \\
0 \text { for }|u|>n .
\end{array}\right.
$$

For a thorough discussion of (2) and of $J$, see [8]. That reference is also recommended in case the reader is unfamiliar with any transformation of (1) employed but not commented upon in the present paper.

Let $L_{+}^{1}$ denote the positive cone in $L^{1}\left(R^{3}\right)$, i.e.

$$
L_{+}^{1}=\left\{f \in L^{1}\left(R^{3}\right) ; \quad f(v) \geqq 0 \text { a.e. } v \in R^{3}\right\},
$$

and set

$$
Q_{n}=Q_{w_{n}}, \mathscr{H} f=\int_{R^{3}} f(v) \log f(v) d v .
$$

In the infinite range case an early paper is [7], a study by Truesdell of an infinite system of moment equations for Maxwellian molecules. The papers [9] by Wang Chang and Uhlenbeck, and [5] by Pao are concerned with the $L^{2}$ eigenvalue problem for the linearized collision operator and inverse $k^{\text {th }}$ power molecules. For $k>3$ we obtained in [3] $L^{1}$ existence results for a weak form of (1). Those results are sketched below and then used to study the asymptotic behaviour of the same Boltzmann equation.

Our proofs in the present paper rely on non-standard arguments and employ the usual notations from that context. We discuss non-standard analysis briefly below but advise the reader to consult [6] and references therein for a full treatment. In [2] our non-standard approach led to a new weaker solution concept derived from a certain non-standard construction. In contrast to that situation, the present main result, contained in Theorem 2 below, is formulated in a standard $L^{1}$ framework. Here the non-standard approach is only a method that allows the use of a previously developed way of proof from the cut-off case, which would be unavailable in that form for the present infinite range forces within the standard framework. On the other hand, as is well known, there is a general procedure translating a non-standard proof of a standard theorem into a standard one. But that standard proof might be neither reasonably short nor particularly natural. An independent proof using standard methods, of the asymptotic behaviour of the Boltzmann equation without cut-off should thus be of interest.

In non-standard analysis proper ordered extensions $* R$ of the field of real numbers are used. ${ }^{*} R$ contains non-zero infinitesimal numbers, and we sometimes 
write $\mathrm{a} \cong 0$ to denote that $\mathrm{a}$ is infinitesimal. The standard part of $a \in^{*} R$ is

$$
\text { st } a={ }^{0} a=\left\{\begin{array}{l}
c \text { if } a \in^{*} R, c \in R, a-c \cong 0 \\
\infty \text { if } a>n(n \in N) \\
-\infty \text { if } a<-n(n \in N) .
\end{array}\right.
$$

The near-standard part of $* R$ is

$$
\text { ns } * R=\left\{x \in *^{*} R ;{ }^{0} x \in R\right\} .
$$

The super-structure on a set $S=S_{0}$ is by definition

$$
V(S)=\bigcup_{n<\infty} S_{n}
$$

where $S_{n+1}$ is the set of all subsets of $\bigcup_{j=0}^{n} S_{j}(n \in N)$. Together with ${ }^{*} R$ a map

$$
*: V(R) \rightarrow V(* R)
$$

is given with

$$
*_{r}=r \quad(r \in R),
$$

and satisfying the transfer principle. This states that if $S_{1}, \ldots, S_{n} \in V(R)$, and $E$ is an elementary statement which is true of $S_{1}, \ldots, S_{n}$ in $V(R)$, then it is true of $* S_{1}, \ldots,{ }^{*} S_{n}$ in $V(* R$ ). (A statement is elementary if it is built up from $\in$ and $=$ using the propositional connectives and bounded quantifiers $\forall x \in y, \exists x \in y$.) Standard sets in $V\left({ }^{*} R\right)$ are $*$-images of sets in $V(R)$. Internal objects in $V\left({ }^{*} R\right)$ are elements of standard sets in $V\left({ }^{*} R\right)$.

Take $n \in *^{*} \backslash N$, and set

$*\left(Q_{n}^{\prime} f, g\right)=\int_{* R^{3} \times R^{3} \times B^{2}}\left[g\left(v_{1}^{\prime}\right)-g\left(v_{1}\right)\right] f \otimes f\left(v_{1}, v_{2}\right) w_{n}\left(v_{1}, v_{2}, u\right)^{*} d v_{1} d v_{2} d u$,

and

$$
\tilde{f}_{0}=* f_{0}+n^{-1} \exp \left(-|v|^{2}\right) .
$$

We next cite several results about (1) proved in [1].

They can by transfer be carried over to our present non-standard setting. The equation

has a solution

$$
\begin{aligned}
\int_{R^{3}} f(t, v) g(t, v)^{*} d v= & \int_{R^{3}} \tilde{f}_{0}(v) g(0, v)^{*} d v+\int_{0}^{t} \int_{R^{3}} f(s, v) D_{s} g(s, v)^{*} d v d s \\
& +\int_{0}^{t}\left(Q_{n}^{\prime} f(s), g(s)\right)^{*} d s \quad\left(g \in \in^{*} C^{1, \infty}\right)
\end{aligned}
$$

$$
f: * R_{+} \rightarrow * L_{+}^{1},
$$

provided $f_{0} \in L_{+}^{1}$. Here

$$
\begin{aligned}
C^{1, \infty}= & \left\{g \in C^{1}\left([0, \infty) \times R^{3}\right) ;|g|_{1}=\sup |g(t, v)|+\sup \left|D_{t} g(t, v)\right|\right. \\
& \left.+\sup \left|\operatorname{grad}_{v} g(t, v)\right|<\infty\right\} .
\end{aligned}
$$


If moreover

$$
\left(1+|v|^{2}\right) f_{0}, f_{0} \log f_{0} \in L^{1}\left(R^{3}\right),
$$

then

$$
\int_{* R^{3}} g(v) f(t, v)^{*} d v=\int_{R^{3}} g(v) f_{0}(v) d v
$$

for

$$
g(v)=1, v,|v|^{2} .
$$

Also $\mathscr{H} f(t)$ is defined for $t>0$ with

$$
\mathscr{H} f(t) \leqq \mathscr{H} f_{0}+\int_{0}^{t} \mathscr{N} f(s)^{*} d s .
$$

Here

$$
\begin{gathered}
\mathscr{N} f=4^{-1} \int_{{ }^{*} R^{3} \times R^{3} \times B^{2}(n)}\left[f \otimes f\left(J_{u}\left(v_{1}, v_{2}\right)\right)-f \otimes f\left(v_{1}, v_{2}\right)\right] w_{i l}\left(v_{1}, v_{2}\right) \\
\log \left[f \otimes f\left(v_{1}, v_{2}\right) / f \otimes f\left(J_{u}\left(v_{1}, v_{2}\right)\right)\right]^{*} d u d v_{1} d v_{2} .
\end{gathered}
$$

The standard weak-limit approach of [3] to existence under infinite range forces does not permit us to go to the limit in (7), whereas in [1] for the cut-off case, that inequality is used in an essential way for the asymptotic studies. But also in the present case with no cut-off we are able to use the approach of [1], if we employ a non-standard setting. That is the main reason for the use of non-standard analysis in this paper.

Since $\mathscr{N} f$ is non-positive, (7) implies that

$$
\mathscr{H} f \leqq \mathscr{H} f_{0} .
$$

By the *-version of Gibbs' lemma

$$
{ }^{0} \mathscr{H} f \geqq \mathscr{H} E_{0} .
$$

Here ${ }^{0}$ denotes the standard part mapping introduced above, and $E_{0}$ is the unique exponential function such that

$$
E_{0}=\exp \left(a|v|^{2}+b \cdot v+c\right)
$$

and for $g(v)$ of $(6)$

$$
\int_{R^{3}} E_{0}(v) g(v) d v=\int_{R^{3}} f_{0}(v) g(v) d v .
$$

For each $t \epsilon^{*} R_{+}$the solution $f$ of (3) defines a mapping

$$
L_{t}: g \rightarrow \int_{* R^{3}}^{0} f(t, v)^{*} g(v) * d v=L_{t}(g),
$$

which is linear from $C_{0}\left(R^{3}\right)$ to $R$ with

$$
\left|L_{t}(g)\right| \leqq \int_{{ }^{*} R^{3}}^{0} f(t, v)^{*} d v \sup _{v \in R^{3}}|g(v)| \leqq \int_{R^{3}} f_{0}(v) d v \sup _{v \in R^{3}}|g(v)| .
$$


It follows that $L_{t}$ defines a measure $\mu_{t}$ on $R^{3}$. Also $\mu_{t}=\mu_{t^{\prime}}$ if $t-t^{\prime} \cong 0$. Under (4) we proved in [3] that

$$
\mu_{t}=F_{t} d v
$$

with $F_{t} \in L_{+}^{1}$, and that

$$
F: R_{+} \rightarrow L_{+}^{1}
$$

is a weak solution of (1) in the sense that

$$
\begin{aligned}
\int_{R^{3}} F_{t}(v) g(t, v) d v= & \int_{R^{3}} f_{0}(v) g(0, v) d v+\int_{0}^{t} \int_{R^{3}} F_{s}(v) D_{s} g(s, v) d v d s \\
& +\int_{0}^{t}\left(Q F_{s}, g(s)\right) d s \quad\left(g \in C^{1, \infty}\right) .
\end{aligned}
$$

Here

$$
(Q f, g)=\int_{R^{3} \times R^{3} \times B^{2}}\left[g\left(v_{1}^{\prime}\right)-g\left(v_{1}\right)\right] f\left(v_{1}\right) f\left(v_{2}\right) w\left(v_{1}, v_{2}\right) d v_{1} d v_{2} d u
$$

In [4] T. Elmroth has shown that certain solutions of (12) generated by a weak $L^{1}$ convergence construction have globally bounded moments of order $s$, if $f_{0}$ has moments of order $s$. This result also holds with an analogous proof for the solutions $F$ above, generated by our non-standard method.

Proposition 1. [4] Assume that for some $s>2$

$$
f_{0} \log f_{0} \in L^{1}\left(R^{3}\right),(1+|v|)^{s} f_{0} \in L_{+}^{1}\left(R^{3}\right) .
$$

Then there exists a constant $C_{f_{0}, s}$ only depending on $s$ and on

such that

$$
\int_{R^{3}}(1+|v|)^{s} f_{0} d v
$$

$$
\int_{R^{3}}(1+|v|)^{s} F(t, v) d v \leqq C_{f_{0}, s} \quad(t>0) .
$$

Here $F$ is the above solution of the Boltzmann equation (12) for $k^{\text {th }}$ power molecules with $k \geqq 5$. Moreover for $g$ of $(6)$

$$
\int_{R^{3}} g(v) F(t, v) d v=\int_{R^{3}} g(v) f_{0}(v) d v
$$

\section{Convergence Towards the Maxwellian}

In this section we study the asymptotic properties of the solution $F$ of (12) constructed in Sect. 1.

Theorem 2. Assume that for some $s>2$

$$
f_{0} \log f_{0} \in L^{1}\left(R^{3}\right),(1+|v|)^{s} f_{0} \in L_{+}^{1} .
$$

Then for $g(v)=(1+|v|)^{s^{\prime}}, 0 \leqq s^{\prime}<s$, the family $(g F(t))_{t>0}$ converges in weak $L^{1}$-sense 
to $g E_{0}$, where $E_{0}$ is the unique exponential function of type (10) which satisfies (11). In the proof the following lemma is used.

Lemma 3. If $s>2$ and the sequence $\left(t_{j}\right)_{j \in N}$ is such that

$$
\sup _{j \in N} \int_{R^{3}}(1+|v|)^{s} F\left(t_{j}, v\right) d v<\infty,
$$

then for

$$
g(v)=(1+|v|)^{s^{\prime}}, 2<s^{\prime}<s,
$$

the sequence $\left(g F\left(t_{j}\right)\right)_{j \in N}$ contains a subsequence converging weakly towards $g G$ for some $G \in L_{+}^{1}$.

Proof. By Dunford-Pettis's theorem the conclusion of the lemma holds, if

a) $\sup _{j \in N} \int_{R^{3}} F\left(t_{j}, v\right)(1+|v|)^{s^{\prime}} d v<\infty$;

b) given $\varepsilon>0$, there exists $\delta>0$, such that

$$
\sup _{j \in N} \int_{A} F\left(t_{j}, v\right)(1+|v|)^{s^{\prime}} d v<\varepsilon \quad \text { if } \quad \int_{A} d v<\delta ;
$$

c) given $\varepsilon>0$, there exists $r_{0}>0$, such that

$$
\sup _{j \in N} \int_{|v| \geqq r_{0}} F\left(t_{j}, v\right)(1+|v|)^{s^{\prime}} \mathrm{dv}<\varepsilon .
$$

Now a) follows from the condition of the lemma, and c) is a consequence of

$$
\int_{|v| \geqq r_{0}} F\left(t_{j}, v\right)(1+|v|)^{s^{\prime}} d v \leqq\left(1+r_{0}\right)^{-s+s^{\prime}} \sup _{j \in N} \int F\left(t_{j}, v\right)(1+|v|)^{s} d v .
$$

For b) we shall use the following estimates.

$$
\begin{aligned}
& \int_{A} F(t, v)(1+|v|)^{s^{\prime}} d v \\
& \quad \leqq(1+r)^{s^{\prime}-s} \int_{|v| \geqq r} F(t, v)(1+|v|)^{s} d v+\int_{A \cap|v|<r} F(t, v)(1+|v|)^{s^{\prime}} d v .
\end{aligned}
$$

For $t=t_{j}$ the first term is bounded by

$$
(1+r)^{s^{\prime}-s} \sup \int_{R^{3}} F\left(t_{j}, v\right)(1+|v|)^{s} d v .
$$

To estimate the second term we notice that for $K>1$

$$
\begin{aligned}
& \int_{A \cap|v|<r} F(t, v)(1+|v|)^{s^{\prime}} d v \leqq \int_{*_{A} \cap|v|<r} f(t, v)(1+|v|)^{s^{\prime} *} d v \\
& \leqq(1 / \log K)(1+r)^{s^{\prime}} \int_{f>K} f(t, v) \log { }^{+} f(t, v)^{*} d v+K(1+r)^{s^{\prime} 0} \int_{*_{A}} * d v .
\end{aligned}
$$


Also

$$
f \log { }^{+} f \leqq f \log f-f \log ^{-} f \leqq f \log f+\exp \left(-|v|^{2}\right)+|v|^{2} f .
$$

Recalling (5) and (8) this gives

$$
\begin{aligned}
& \int_{A} F\left(t_{j}, v\right)(1+|v|)^{s^{\prime}} d v \leqq(1+r)^{s^{\prime}-s} \sup _{j \in N} \int_{R^{3}} F\left(t_{j}, v\right)(1+|v|)^{s} d v \\
& \quad+K(1+r)^{s^{\prime}} \int_{A} d v+(1 / \log K)(1+r)^{s^{\prime}}\left[\mathscr{H} f_{0}+\int_{R^{3}} \exp \left(-|v|^{2}\right) d v\right. \\
& \left.\quad+\int_{R^{3}} f_{0}(v)|v|^{2} d v\right] .
\end{aligned}
$$

From here b) is immediate.

Proof of Theorem 2. With $f$ our non-standard solution of (3) there is by (7) and (9) an increasing non-standard sequence $\left(t_{j}\right)_{j \in N}$, such that

$$
\lim _{j \rightarrow \infty}{ }^{0} \mathscr{N} f\left(t_{j}\right)=0 .
$$

For $s^{\prime}<s$, we can take a subsequence $\left(t_{j_{v}}\right)_{v \in N}$, such that $\left((1+|v|)^{s^{\prime}} F\left(t_{j_{v}}\right)\right)_{v \in N}$ converges in weak $L^{1}$ sense to some $L_{+}^{1}$ function $(1+|v|)^{s^{\prime}} E$. This is possible by Lemma 3 and Proposition 1. Rename for simplicity the subsequence to $\left(t_{j}\right)_{j \in N}$, and notice that in particular

$$
\int_{R^{3}} g(v) E(v) d v=\lim _{j \rightarrow \infty} \int_{R^{3}} g(v) F\left(t_{j}, v\right) d v=\int_{R^{3}} g(v) f_{0}(v) d v
$$

for $g$ of (6).

Define

for functions

$$
\Delta G\left(v_{1}, v_{2}, u\right)=G \otimes G\left(J_{u}\left(v_{1}, v_{2}\right)\right)-G \otimes G\left(v_{1}, v_{2}\right)
$$

$$
G: R^{3} \times R^{3} \rightarrow R
$$

We shall next prove by contradiction that

$$
\Delta E\left(v_{1}, v_{2}, u\right)=0 \text { for a.e. }\left(v_{1}, v_{2}, u\right) \in R^{3} \times R^{3} \times R^{2} .
$$

So let us suppose that $\Delta E \neq 0$ on a set of positive measure. Then there is a bounded, measurable set $A \subset R^{3} \times R^{3} \times R^{2}$ of measure $m>0$,

$$
A \subset\left\{\left(v_{1}, v_{2}, u\right) ;\left|v_{1}\right|+\left|v_{2}\right|+|u| \leqq r_{0}\right\}
$$

for some real $r_{0}$, and

$$
\int_{A} \Delta E\left(v_{1}, v_{2}, u\right) w\left(v_{1}, v_{2}\right) d u d v_{1} d v_{2}=C_{0}
$$

for some real $C_{0}$ with $0<C_{0}<1$. This will below turn out to contradict (13).

Let $K>1$ be given together with an arbitrary subset $A^{\prime} \subset A$ of Lebesgue measure

$$
\int_{A^{\prime}} d v_{1} d v_{2} d u<K^{-2}
$$


Then

$$
\begin{aligned}
& \int_{A^{\prime}} \Delta F\left(t, v_{1}, v_{2}, u\right) w\left(v_{1}, v_{2}\right) d u d v_{1} d v_{2} \\
& \leqq 2 r_{0} \int_{* J\left(A^{\prime}\right)}^{0} f \otimes f\left(t, v_{1}, v_{2}, u\right) * d u d v_{1} d v_{2} \\
& \leqq 2 r_{0}\left(K^{-1}+(1 / \log K) 2 \pi r_{0}^{2}\left[\mathscr{H} f_{0}+\int_{R^{3}} \exp \left(-|v|^{2}\right) d v\right.\right. \\
& \left.\left.+\int_{R^{3}} f_{0}(v)|v|^{2} d v\right] \int_{R^{3}} f_{0}(v) d v\right) .
\end{aligned}
$$

The proof of the last estimate is similar to that of b) in Lemma 3 .

By (16) there is a standard $K_{0}$, such that

$$
\int_{A^{\prime}} \Delta F\left(t, v_{1}, v_{2}, u\right) w\left(v_{1}, v_{2}\right) d u d v_{1} d v_{2}<C_{0} / 4
$$

if $A^{\prime} \subset A, t>0$, and $\int_{A^{\prime}} d v_{1} d v_{2} d u<\pi r_{0}^{2} / K_{0}^{2}$. The subset of $A$ with

$$
F \otimes F\left(t, v_{1}, v_{2}\right)>K_{0}^{2}\left(\int_{R^{3}} f_{0}(v) d v\right)^{2}=C_{1}
$$

has measure smaller than $\pi r_{0}^{2} / K_{0}^{2}$, since

$$
\int_{R^{3}} F(t, v) d v=\int_{R^{3}} f_{0}(v) d v .
$$

This implies together with the discussion preceding (14) that there is an index $j_{0}$, and given $j>j_{0}$ a set $A_{j} \subset A$ of measure larger than $m-\pi r_{0}^{2} / K_{0}^{2}$, such that

on $A_{j}$, and

$$
F \otimes F\left(t_{j}, v_{1}, v_{2}\right) \leqq K_{0}^{2}\left(\int_{R^{3}} f_{0}(v) d v\right)^{2}
$$

$$
\begin{aligned}
& \int_{*_{A_{J}}^{0}} \Delta f\left(t_{j}, v_{1}, v_{2}, u\right) w\left(v_{1}, v_{2}\right) * d u d v_{1} d v_{2} \\
& \quad=\int_{A_{J}} \Delta F\left(t_{j}, v_{1}, v_{2}, u\right) w\left(v_{1}, v_{2}\right) d u d v_{1} d v_{2}>C_{0} / 2 .
\end{aligned}
$$

Set $\Delta^{+} f=\max (0, \Delta f)$. By a proof in [1] carried over to our present non-standard setting, there is a standard $\varepsilon>0$ and for each $j>j_{0}$ a partition $* A_{j}=A_{j 1} \cup A_{j 2}$, such that

$$
\begin{aligned}
{ }^{0} \mathcal{N} f\left(t_{j}\right) \leqq & -\left(4 m C_{1} r_{0}\right)^{-1}\left(\int_{A_{j 1}} \Delta^{+} f\left(t_{j}\right) w^{*} d u d v_{1} d v_{2}\right)^{2} \\
& -\log (1+\varepsilon)^{0} \int_{A_{j 2}} \Delta^{+} f\left(t_{j}\right) w^{*} d u d v_{1} d v_{2} \\
\leqq & -\min \left(\left(4 m C_{1} r_{0}\right)^{-1}, \log (1+\varepsilon)\right) C_{0}^{2} / 16
\end{aligned}
$$


This contradicts (13) and so (15) holds a.e.. But (15) implies that $E=E_{0}$, with $E_{0}$ determined by (10) and (11) (cf [1]).

We shall finally, again by contradiction prove that $(1+|v|)^{s^{\prime}} E_{0}$ for $s^{\prime}<s$ is the weak $L^{1}$ limit of $(1+|v|)^{s^{\prime}} F(t)$, when $t \rightarrow \infty$. So let us suppose the opposite. Then there is a sequence $\left(t_{j}\right)_{j \in N}, \lim _{j \rightarrow \infty} t_{j}=\infty$, with

$$
\lim _{j \rightarrow \infty}(1+|r|)^{s^{\prime}} F\left(t_{j}\right)=(1+|v|)^{s^{\prime}} E^{\prime} \neq(1+|v|)^{s^{\prime}} E_{0},
$$

the limit taken in weak $L^{1}$ sense, as well as a function $g \in C^{1, \infty}$, such that

$$
\int_{R^{3}} E^{\prime}(v) g(v) d v \neq \int_{R^{3}} E_{0}(v) g(v) d v
$$

We proved in [3] that

$$
\begin{aligned}
& \left|\int_{R^{3}}\left(F\left(t_{2}, v\right)-F\left(t_{1}, v\right)\right) g(v) d v\right| \\
& \leqq|g|_{1}\left|t_{2}-t_{1}\right|\left[\int_{R^{3}} f_{0}(v) d v+C\left(\int_{R^{3}}\left(1+|v|^{2}\right) f_{0}(v) d v\right)^{2}\right] .
\end{aligned}
$$

And so there is $h>0$, such that for $t>0$

$$
\left|\int_{R^{3}}(F(t+h, v)-F(t, v)) g(v) d v\right|<\left|\int_{R^{3}}\left(E^{\prime}(v)-E_{0}(v)\right) g(v) d v / 2\right| .
$$

By (7) and (9) for some finite $C$

$$
0 \leqq \int_{0}^{h} \sum_{j \leqq \bar{n}}-\mathcal{N} f\left(t_{j}+s\right)^{*} d s<C, \bar{n} \in N .
$$

Since $\mathscr{N} f \leqq 0$, there is some $s_{0}, 0<s_{0}<h$, with

$$
\lim _{N \in J \rightarrow \infty}^{0} \mathscr{N} f\left(t_{j}+s_{0}\right)=0 .
$$

As in the case of (13) above, from here we conclude the existence of a subsequence $\left(t_{j_{v}}\right)_{v \in N}$ with the weak $L^{1}$ limit of $F\left(t_{j_{v}}+s_{0}\right)_{v \in N}$ equal $E_{0}$. Thus

$$
\lim _{v \rightarrow \infty} \int_{R^{3}}\left(F\left(t_{j_{v}}+s_{0}, v\right)-F\left(t_{j_{v}}, v\right)\right) g(v) d v=\int_{R^{3}}\left(E_{0}(v)-E^{\prime}(v)\right) g(v) d v \neq 0 .
$$

This contradicts (17), and so $(1+|v|)^{s^{\prime}} E_{0}$ is the weak $L^{1}$ limit of $(1+|v|)^{s^{\prime}} F(t)$ when $t \rightarrow \infty$.

Remark. By Theorem 2, any moment of $F$ of order less than $s$ converges towards the same moment of $E_{0}$, when $t \rightarrow \infty$. For $k=5$ we obtained a better result in [3]. As discussed there, the moments in the case $k=5$ satisfy Truesdell's moment equations, and converge exponentially to those of $E_{0}$. 


\section{References}

1. Arkeryd, L.: On the Boltzmann equation. Arch. Rat. Mechs. Anal. 45, 1--34 (1972)

2. Arkeryd, L.: A non-standard approach to the Boltzmann equation. Arch. Rat. Mechs. Anal. 77, $1-10(1981)$

3. Arkeryd, L.: Intermolecular forces of infinite range and the Boltzmann equation. Arch. Rat. Mechs. Anal. 77, 11-21 (1981)

4. Elmroth, T.: Global boundedness of moments of solutions of the Boltzmann equation for infinite range forces. Arch. Rat.

5. Pao, Y. P.: Boltzmann collision operator with inverse-power intermolecular potentials. Commun. Pure Appl. Math. 407-428, 559-581 (1974)

6. Stroyan, K. D., Luxemburg, W. A. J.: Introduction to the theory of infinitesimals. New York: Academic Press 1976

7. Truesdell, C.: On the pressures and the flux of energy in a gas according to Maxwell's kinetic theory, II. J. Rat. Mechs. Anal. 5, 55-128 (1956)

8. Truesdell, C., Muncaster, R. G.: Fundamentals of Maxwell's kinetic theory of a simple monatomic gas. New York: Academic Press 1980

9. Wang Chang, C. S., Uhlenbeck, G. E.: On the propagation of sound in Monatomic Gases. Eng. Res. Inst. Rep. M999, Univ. of Michigan 1952

Communicated by J. L. Lebowitz

Received October 1, 1981; in revised form February 2, 1982 